Comparing intentions to reduce substance use and willingness to seek help among transgender and cisgender participants from the global drug survey

Dean Connolly ${ }^{1 \star}$, Emma Davies ${ }^{2}$, Michael Lynskey ${ }^{3}$, Monica Barratt ${ }^{4}$, Larissa Maier ${ }^{5}$, Jason Ferris ${ }^{6}$, Adam Winstock ${ }^{7}$ and Gail Gilchrist ${ }^{3}$

${ }^{1}$ IoPNN King's College London; ${ }^{2}$ Oxford Brookes University; ${ }^{3}$ IoPPN King's College London; ${ }^{4}$ RMIT University; ${ }^{5}$ University of California; ${ }^{6}$ The University of Queensland and ${ }^{7}$ University College London ${ }^{*}$ Corresponding author.

doi: 10.1192/bjo.2021.654

Aims. To describe and compare psychoactive substance misuse help-seeking among transgender (trans) and cisgender (cis) participants from a large multi-national cross-sectional survey.

Background. Trans people experience stressors related to their minority status which have been associated with increased rates of psychoactive substance use and related harm. Despite this, there is a paucity of evidence relating to the treatment needs of trans people who use psychoactive substances, beyond a small body of literature describing a culture of transphobic hostility in general substance misuse services. This paper aims to describe and compare psychoactive substance misuse help-seeking among trans and cis participants from a large multi-national cross-sectional survey.

Method. Over 180,000 participants, recruited from the world's largest annual survey of drug use - the Global Drug Survey (GDS) - during 2018 and 2019, reported use of a range of psychoactive substances in the preceding 12 months. Five gender groups $(118,157$ cis men, 64,319 cis women, 369 trans men, 353 trans women and 1,857 non-binary people) were compared, using Chi-square and z-tests with Bonferroni correction, on items relating to the desire to use less psychoactive substances and the need to seek help to achieve this. Respondents from GDS 2018 were also assessed for substance dependence. Binary logistic regression was used to compare gender groups on self-reported substance dependence to frame the help-seeking analyses.

Result. Trans respondents $(n=1,710)$ to GDS 2018 were significantly more likely than cis respondents to report use of illicit substances $(\mathrm{OR}=1.66-2.93)$ and dependence on cannabis $(\mathrm{OR}=$ $2.39)$, alcohol $(\mathrm{OR}=3.28)$ and novel psychoactive substances $(\mathrm{OR}=4.60)$. In the combined GDS 2018 and 2019 dataset, there were no significant differences between trans $(n=2,579)$ and cis $(n=182,476)$ participants on the desire to reduce substance use. However, among those who did report wanting to use less, nonbinary people and trans women were most likely to want help to achieve this.

Conclusion. Trans respondents reported a greater need for help with reducing substance use than cis respondents. Given the deficit of specialist services for psychoactive substance users who are trans, there is a need for a more thorough understanding of the barriers and facilitators to their engagement in general substance misuse services. In the interim, substance misuse service providers require education about gender minority status to help meet the needs of trans clients.

\section{Prevalence and correlates of substance use among transgender adults: a systematic narrative review}

Dean Connolly ${ }^{1 \star}$ and Gail Gilchrist ${ }^{2}$

${ }^{1}$ IoPNN King's College London and ${ }^{2}$ IoPPN King's College London ${ }^{\star}$ Corresponding author.

doi: 10.1192/bjo.2021.655
Aims. To understand the prevalence, patterns and correlates of substance use among transgender adults.

Background. Minority stress theories suggest that the increasing rates of discrimination experienced by transgender people are precipitants of substance use. This is likely exacerbated by an inadequate provision of trans-inclusive substance misuse services. However, the exclusion of transgender people from the general substance misuse literature makes it difficult to determine the extent to which gender minority status influences substance use. A systematic review was undertaken to better understand the prevalence, patterns and correlates of substance use among this group.

Method. In accordance with the PRISMA guidance, a literature search was conducted to 29th May 2019 on PubMed, PsycINFO, EMBASE and Global Health databases. Primary quantitative studies, published in the English language, that reported the prevalence, patterns or correlates/risk factors of substance use by trans people were included, with no restriction on methodological design.

Result. 651 unique records were identified by the search and 40 studies were included in the synthesis. While there was some suggestion of excess risk of substance use among trans people, there was insufficient evidence to estimate prevalence or quantify the risk of substance use among transgender people, relative to a cisgender population. However, this review identified several gender minority related correlates of substance use which are of relevance to clinicians working with transgender patients, including transphobic discrimination or violence, unemployment and sex work, gender dysphoria, high visual gender non-conformity and intersectional sexual minority status.

Conclusion. There are currently significant gaps in the trans substance use literature, relating to the disproportionate investigation of transgender women with multiple intersectional disadvantages, who are not representative of the wider trans community. However, there is sufficient evidence to recommend screening for substance use when individuals report high levels of gender minority stress and to consider the use of integrated trauma-informed psychosocial interventions when managing problematic substance use in the transgender adult.

\section{A clinical inventory of moderators of tic severity in Tourette's Syndrome}

Francesca Conti ${ }^{1 \star}$ and Himanshu Tyagi ${ }^{2}$

${ }^{1}$ UCL Division of Psychiatry and ${ }^{2}$ UCL Queen Square Institute of Neurology, The National Hospital for Neurology and Neurosurgery ${ }^{\star}$ Corresponding author.

doi: $10.1192 /$ bjo.2021.656

Aims. Changes in the severity of tics in Tourette's syndrome (TS), as seen with variations in the intensity or frequency of tics, can be moderated by a variety of independent factors such as external or internal stimuli. Identifying such moderators has important clinical implications as it can aid clinicians in adjusting interventions. Hence, based on our previous review of tic-severity moderators, we developed a clinical inventory of moderating variables for motor and vocal tics for inclusion in the new version of the Queen Square Proforma for Tourette's Syndrome to aid initial assessments in the National Tourette Syndrome Service's Outpatient Clinic for Adults.

Method. A review of tic-severity moderators was previously carried out by the authors to investigate the kinds of moderators and their worsening, improving or neutral effects on tic severity. Based on this a semantic thematic analysis of the identified 